Theoretical Physics, Astrophysics and Cosmology

Vol. 4, No 2, p. 10 - 16, v1, 5 October 2009

Online: TPAC: $3200-014$ v2, 18 September 2012
ISSN 1819-9135; ISSN 1991-3919 (e)

(C) 2009, 2012 CTPA. All rights reserved. DOI: $10.9751 /$ TPAC.3200-014

\title{
The theory of stochastic space-time. 2. Quantum theory of relativity ${ }^{1}$
}

\begin{abstract}
Zahid Zakir ${ }^{2}$
Abstract

Nelson's stochastic mechanics is derived as a consequence of the basic physical principles such as the principle of relativity of observations and the invariance of the action quantum. The unitary group of quantum mechanics is represented as the transformations of the systems of perturbing devices. It is argued that the physical spacetime has a stochastic nature and that quantum mechanics in Nelson's formulation correctly describes this stochasticity.
\end{abstract}

PACS: 04.20.Cv, 03.65.Ta, 05.40.Jc, 04.62.+v

Key words: stochastic mechanics, quantum fluctuations, measurements

\section{Content}

Introduction

1. Canonical transformations as transformations of the systems of unperturbing devices ............11

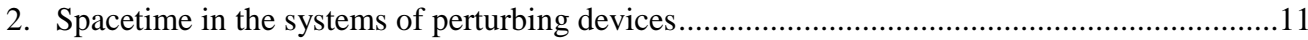

3. Relativity of perturbing observations and transformations of SPD .........................................12

4. The principle of constancy of action quantum and the diffusion coefficient ..........................13



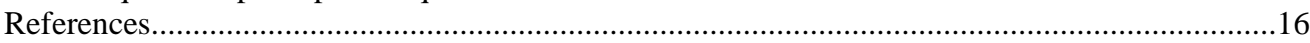

\section{Introduction}

A physical description is based on the analysis of results of observations; however, the laws of physics should not depend on methods of observations and a choice of an observer. This condition we shall name as the principle of relativity of observations, and consider as a general principle to which must satisfy all physical theories. In the paper we shall consider some measurement procedures and mathematical structures, through which this general principle is manifested in classical and quantum physics.

Particularly, the canonical transformations of Hamiltonian dynamics in some cases describe transformations of systems of unperturbing devices, the unitary transformations of Hilbert space of states in quantum physics can be represented as transformations of the systems of perturbing devices. This means that one can take as the first principles of quantum mechanics the such physical principles as the relativity under the systems of measuring devices and the invariance of the fundamental constants - the action quantum $\hbar$ and the light velocity $c$, which lead to a stochastic geometry [1,2] of the physical spacetime [3].

\footnotetext{
${ }^{1}$ The preprint of the paper has been presented in 1999 (revised 2003): Zakir Z. arXiv:hep-th/9901013

${ }^{2}$ Centre for Theoretical Physics and Astrophyics, Tashkent, Uzbekistan; zahidzakir@theor-phys.org
} 
In the preceding paper [4] some consequences of stochastic treatment of gravitation has been considered. In the present paper it will be shown that this treatment can be derived from some general physical principles.

\section{Canonical transformations as transformations of the systems of unperturbing devices}

In the classical physics the system of coordinates have been constructed by using the systems of devices allowing one to measure complete sets of dynamical variables near each point of space. There it has been implicitly supposed, that the basic equations of physics do not depend on a choice of the system of unperturbing devices. We shall name this statement as the principle of relativity of unperturbing observations (PRUO).

If an object in one a system of unperturbing devices is described by a full set of dynamical variables - a generalized coordinate and momentum $(q, p)$ and on the second system by another set of coordinates and momentum $(P, Q)$ at the same conditions, then according to this principle the equations of motion should not depend on the transition from the first pair of variables to the second one.

We see that the definition of transformations of the systems of unperturbing devices is the same as the definition of the canonical transformations, and therefore, we can suppose that the canonical invariance of the equations of motion of classical physics is expression of the principle of relativity of unperturbing observations. This treatment allows one to describe the states of mechanical objects by means of the systems of unperturbing devices in a phase space with the symplectic structure, where the principle of relativity of unperturbing observations is represented across the canonical group of symmetry.

From this point of view, the applicability of the Hamiltonian dynamics to various physical structures partly testifies that their dynamical variables can be measured by the system of unperturbing devices, or they can be reduced to such variables. This interpretation partly explains the universality of the Hamiltonian structures not only in the classical mechanics, but in other fields of physics also.

\section{Spacetime in the systems of perturbing devices}

The existence an action quantum, equal to the Planck constant $\hbar$, requires extending the principle of relativity of observations to the systems of perturbing devices (SPD). Further we shall convince, that such extension is possible, and moreover, that the quantum theory can be considered as its result. Here we shall consider the change of the structure of space and time in SPD.

Let we have a system of perturbing devices as a set of classically described measurement devices near each point of Euclidean space. Let during the measurements of coordinates and times of classical particles of small mass, the particles scatter on devices at many points of their trajectories. Then, in the limiting case of very small times between measurements, the trajectories of such particles will be similar to Brownian trajectories, and the classical mechanics must be replaced by the theory of Brownian motion. The system of perturbing devices here plays a role of an environment with some diffusion coefficient and the observables should be defined in a statistical ensemble of measurements. Instead of the definite coordinates and momentum of the particles, here we deal with the probability densities and the transition probability densities.

Two kinds of Brownian processes are known - the usual dissipative diffusion and nondissipative (Nelson's) diffusion [1]. The examples of the systems of perturbing devices 
with dissipative diffusion are the Wilson and bubble chambers in which a high energy particle interacts with atoms of the medium along the trajectory and loses the energy.

At Nelson's diffusion the energy of an ensemble of particles is conserved and the equations of diffusion are reversible in time in contrary to the irreversible dissipative diffusion. Here we consider the construction of such conservative systems of devices, in which the motion of particles represents Nelson's diffusion [1]. This is a set of large number massive screens with infinite number slits on each screen [5]. Let the slits have massive shutters, which must rapidly open and close the slits during very short time. At the opening and closing of the shutters the sample particles scatter on them. As the result, the energy and momentum of the particles may change sufficiently, while the mean energy and momentum of the massive shutters and screens do not change at such scatterings. Therefore, the tangent components of the momentum and kinetic energy of the scattering particles change stochastically but their mean values remain unchanged.

Here a physical reason for the conservative behavior is the fact, that all elements of the measuring devices are massive (macroscopic) objects, essentially exceeding the masses of the sample particles. At such ratio of the masses, it is possible to consider the collisions of the classical particles of small mass with very massive devices as absolutely elastic (on a rest frame of the shutter). The energy conservation of the ensemble of particles leads to the temporal reversibility of their equations of motions.

Thus in SPD the structure of spacetime becomes stochastic and the Galilean (or Minkowski) geometry of spacetime should be replaced by the stochastic geometry. In the dissipative SPD this is stochastic spacetime with Wiener's measure, and in nondissipative SPD - spacetime with Nelson's measure.

\section{Relativity of perturbing observations and transformations of SPD}

In classical mechanics with unperturbing devices it is insufficient how many screens there are between two spatial points at particle's trajectory. However, if we take into account the scattering of the particle on the screens with moving shutters, then a number of scatterings becomes sufficient for the final probability density of such observed particles. At the increasing of the number $N$ of the screens between initial and final positions, the temporal intervals between the scatterings of the particles on neighbor shutters decrease, and at $N \rightarrow \infty$ we have $\Delta t \rightarrow 0$. In fact, this is nothing but as some transformation of the system of perturbing devices, and the physically interesting variables are those which tend to finite values at the such increasing of $N$.

Here we consider such transformations of SPD and some conditions required by the principle of relativity of observations. An ensemble of particles in SPD is described by a probability density $\rho(\mathbf{x}, t)$ and a transition probability density $p\left(\mathbf{x}^{\prime}, t^{\prime} ; \mathbf{x}, t\right)$. In fact, there are two families of transition probabilities $p_{ \pm}$, where $p_{+}\left(\mathbf{x}, t ; \mathbf{x}_{0}, t_{0}\right)$, describes the direct in time transition probabilities $t>t_{0}$, while $p_{-}\left(\mathbf{x}, t ; \mathbf{x}_{0}, t_{0}\right)$ describes the backward in time transition probabilities $t<t_{0}$. It is clear that in the conservative diffusion both types must appear in the symmetric form. In the particular case of Nelson's kinematics these two types of transition probabilities can be reduced to one a function $S(\mathbf{x}, t)$ and a diffusion coefficient $v$ [1] (see review [2]).

A given SPD differs from another one only by the transition probability densities $p_{ \pm}\left(\mathbf{x}, t ; \mathbf{x}_{0}, t_{0}\right)$ describing the temporal evolution of $\rho(\mathbf{x}, t)$. For $\rho(\mathbf{x}, t)$ on the first SPD one has: 


$$
\rho(\mathbf{x}, t)=\int p_{ \pm}\left(\mathbf{x}, t ; \mathbf{x}_{0}, t_{0}\right) \rho\left(\mathbf{x}_{0}, t_{0}\right) d \mathbf{x}_{0},
$$

Here $\rho^{\prime}$ and $p_{ \pm}^{\prime}$ transform as:

$$
\begin{gathered}
\rho^{\prime}(\mathbf{x}, t)=B(\mathbf{x}, t) \rho(\mathbf{x}, t)=(1+\delta B) \rho=\rho+\delta \rho, \\
p_{ \pm}^{\prime}\left(\mathbf{x}, t ; \mathbf{x}_{0}, t_{0}\right)=B(\mathbf{x}, t) p_{ \pm}\left(\mathbf{x}, t ; \mathbf{x}_{0}, t_{0}\right) B^{-1}\left(\mathbf{x}_{0}, t_{0}\right)= \\
=p_{ \pm}+\left(\delta B p_{ \pm}-p_{ \pm} \delta B_{0}\right)=p_{ \pm}+\delta p_{ \pm},
\end{gathered}
$$

where $B(\mathbf{x}, t)$ is the operator for the transformations of $\rho(\mathbf{x}, t)$ at the changings of SPD.

The probability conservation conditions:

$$
\int \rho(\mathbf{x}, t) d \mathbf{x}=\int \rho^{\prime}(\mathbf{x}, t) d \mathbf{x}=\int B(\mathbf{x}, t) \rho(\mathbf{x}, t) d \mathbf{x}=1,
$$

lead for small variations:

$$
\begin{gathered}
\int \delta \rho(\mathbf{x}, t) d \mathbf{x}=0, \\
\int \delta B(\mathbf{x}, t) \rho(\mathbf{x}, t) d \mathbf{x}=\langle\delta B\rangle=0,
\end{gathered}
$$

since only the spatial distributions of $\rho(\mathbf{x}, t)$ and $\delta B$ change at such local deformations.

The velocities and diffusion coefficients of particles in SPD are defined as the conditional expectations

$$
\begin{gathered}
\int d \mathbf{x}_{0} p_{ \pm}\left(\mathbf{x}, t ; \mathbf{x}_{0}, t_{0}\right)\left(\mathbf{x}-\mathbf{x}_{0}\right)= \pm \mathbf{b}_{ \pm}(\mathbf{x}, t) \Delta t, \\
\int d \mathbf{x}_{0} p_{ \pm}\left(\mathbf{x}, t ; \mathbf{x}_{0}, t_{0}\right)\left(x_{i}-x_{i 0}\right)\left(x_{j}-x_{j 0}\right)= \pm 2 n_{ \pm}(\mathbf{x}, t) \delta_{i j} \Delta t,
\end{gathered}
$$

which transform at the SPD transformations as:

$$
\begin{gathered}
\lim _{\Delta t \rightarrow 0} \int d \mathbf{x}_{0} B(\mathbf{x}, t) p_{ \pm}\left(\mathbf{x}, t ; \mathbf{x}_{0}, t_{0}\right) B^{-1}\left(\mathbf{x}_{0}, t_{0}\right)\left(\mathbf{x}-\mathbf{x}_{0}\right)= \pm \mathbf{b}_{ \pm}^{\prime}(\mathbf{x}, t) d t \\
\lim _{\Delta t \rightarrow 0} \int d \mathbf{x}_{0} B(\mathbf{x}, t) p_{ \pm}\left(\mathbf{x}, t ; \mathbf{x}_{0}, t_{0}\right) B^{-1}\left(\mathbf{x}_{0}, t_{0}\right)\left(x_{i}-x_{i 0}\right)\left(x_{j}-x_{j 0}\right)= \pm 2 n_{i j \pm}^{\prime}(\mathbf{x}, t) d t .
\end{gathered}
$$

\section{The principle of constancy of action quantum and the diffusion coefficient}

In classical mechanics it can be constructed SPD with arbitrary small perturbations, and for a classical particle observing by SPD the conditional expectation of the action function $\Delta A_{ \pm}$vanish for infinitesimal temporal intervals:

$$
\lim _{\Delta t \rightarrow 0} \int d \mathbf{x}_{0} p_{ \pm}\left(\mathbf{x}, t ; \mathbf{x}_{0}, t_{0}\right) \Delta A_{ \pm}\left(\mathbf{x}, t, \mathbf{x}_{0}, t_{0}\right)=0,
$$

which means that:

$$
\lim _{\Delta t \rightarrow 0} \int d \mathbf{x}_{0} p_{ \pm}\left(\mathbf{x}, t ; \mathbf{x}_{0}, t_{0}\right)\left[\frac{m\left(\mathbf{x}-\mathbf{x}_{0}\right)^{2}}{ \pm \Delta t}\right]=0 .
$$

Since the classical mechanics is not exact theory for the microscopic phenomena, in the general case one must to take into account the existence of the action quantum (Planck's constant) $\hbar$ which should be invariant under the transformations of SPD. The last statement we shall call as the principle of constancy of action quantum [3]. Particularly, if the conditional expectation of $\Delta A_{ \pm}$is equal to $\hbar$ in one of SPD, then it should be equal to $\hbar$ in all other SPD. So, we have the expression: 


$$
\lim _{\Delta t \rightarrow 0} \int d \mathbf{x}_{0} p_{ \pm}\left(\mathbf{x}, t ; \mathbf{x}_{0}, t_{0}\right)\left[\frac{m\left(\mathbf{x}-\mathbf{x}_{0}\right)^{2}}{ \pm \Delta t}\right]=\hbar
$$

or in the SPD transformed form:

$$
\lim _{\Delta t \rightarrow 0} \int d \mathbf{x}_{0} B(\mathbf{x}, t) p_{ \pm}\left(\mathbf{x}, t ; \mathbf{x}_{0}, t_{0}\right) B^{-1}\left(\mathbf{x}_{0}, t_{0}\right)\left[\frac{m\left(\mathbf{x}-\mathbf{x}_{0}\right)^{2}}{ \pm \Delta t}\right]=\hbar .
$$

We see that, as the consequence of this principle, the conditional expectation $E\left[d A_{ \pm} \mid \mathbf{x}(t)\right]$ don't vanish at $\Delta t \rightarrow 0$. As the result, we obtain the SPD covariant formula for the mean square values of particle's coordinates:

$$
\lim _{\Delta t \rightarrow 0} \int d \mathbf{x}_{0} p_{ \pm}^{\prime}\left(\mathbf{x}, t ; \mathbf{x}_{0}, t_{0}\right)\left(\mathbf{x}-\mathbf{x}_{0}\right)^{2}= \pm \frac{\hbar}{m} d t= \pm 2 v d t,
$$

where the diffusion coefficient $v=\hbar / 2 m$ is exactly the same as in Nelson's stochastic formulation of quantum mechanics [1]. But in the stochastic mechanics the value of diffusion coefficient has been given equal to $v=\hbar / 2 m$ by hand, whereas in the present treatment this formula directly follows from the physically clear first principles.

Since for the SPD with such invariant diffusion coefficients $n_{i j \pm}^{\prime}= \pm 2 v \delta_{i j}$ the osmotic velocities $\mathbf{u}=\left(\mathbf{b}_{+}-\mathbf{b}_{-}\right) / 2$ can be expressed across $\rho(\mathbf{x}, t)$, the SPD transformations can be reduced to the transformations of $\rho(\mathbf{x}, t)$ and $\mathbf{v}=\left(\mathbf{b}_{+}+\mathbf{b}_{-}\right) / 2$ only:

$$
\begin{aligned}
& \delta \mathbf{u}=v \delta\left(\frac{\nabla \rho}{\rho}\right), \\
& \delta \mathbf{v}=\frac{1}{m} \nabla(\delta S),
\end{aligned}
$$

where $S(\mathbf{x}, t)$ is some function, introduced instead of $\mathbf{v}(\mathbf{x}, t)$ by the expression:

$$
m \mathbf{v}=\nabla S,
$$

and which can be derived from an initial Lagrangian after the special SPD transformations [6].

Finally, we have a functional "phase space" by the canonical pair $(\rho, S)$ for particle's motion in SPD, and the corresponding algebra of observables [7] which are exactly equivalent to the Hilbert space of states and operator algebra of the ordinary quantum mechanics.

\section{The quantum principle of equivalence}

The mass parameter $m$ in the diffusion coefficient of SPD $v_{d}=\hbar / 2 m_{i n}$, considered in the previous Section, is the inertial mass $m_{i n}$ determined by the kinetic term of an action function describing the scattering of particles on SPD.

In Nelson's stochastic mechanics we also have the diffusion coefficient with the same inertial mass $v_{s}=\hbar / 2 m_{i n}$. There a classical sample particle with the inertial mass $m_{i n}$ freely moves in the stochastic space and the process represents the inertial motion in form of the conservative diffusion on some background. 
In quantum mechanics, rewritten in terms of the stochastic processes, we have the effective diffusion coefficient $v_{q}=\hbar / 2 m_{q}$, where $m_{q}$ is some mass parameter, determining the fluctuations of coordinates of quantum particles in flat and regular Galilean space and time [8]. As it was shown from analysis of the Lamb shift data, the quantum diffusion mass $m_{q}$ is equal to the inertial mass with high accuracy [8]:

$$
\left(m_{\text {in }}-m_{q}\right) / m_{\text {in }} \leq 10^{-13} \text {. }
$$

Therefore, two kinds of masses of the particle are equal to each other:

$$
m_{i n}=m_{q} \text {, }
$$

and this fact is very important for the understanding of a geometrical nature of quantum phenomena.

Firstly this means the equivalence of the motion of the classical particle on SPD in the ordinary smooth space to the motion of the classical particle in the stochastic space with unperturbing devices. This fact means also the equivalence of the transition to SPD and the quantum mechanical description (quantization) of the motion of the classical particle.

We can demonstrate this situation in the simple double slit experiment. Let we have a source and a detector of the particles and there exists between them a screen with two slits. Let the particles, emitted by the source and penetrated across the slits, have been registered by the detectors. After the repeating the experiment many times, an observer obtains the interference picture on the detectors. The observer, which has only photographic plate with interference picture, cannot distinguish three interpretations:

a) Space is empty and Euclidean, but the particles have "quantum" properties (wave function) leading to the interference. This is the quantum mechanical interpretation;

b) Space is empty and stochastic, and the motion of the classical particles on this background leads to the interference. This is the interpretation of the stochastic mechanics;

c) Space is smooth and Euclidean, but it not empty, and there exist large number of devices placed near each point of space. The classical particles interact with this medium of devices, and as the result, the observer detects the interference. This treatment based on the thought experiment illustrating the principle of relativity of observations.

Further we will call this fact as the quantum principle of equivalence [3]. Can we conclude from these statements that quantum mechanics is the stochastic geometry of spacetime and that the stochastic mechanics is a true physical formulation of quantum mechanics? Is the physical spacetime stochastic?

For the answer to these questions, let us remind an analogue with Einstein's proof of a geometrical nature of gravitation. From the equality of the inertial and gravitational masses in general relativity, one has indistinguishability for the observer of three treatments in the explaining of acceleration of a sample particle:

a) spacetime is Euclidean, the reference frame is inertial, but there exist a gravitational field with the potential $\phi$ (the field treatment);

b) spacetime is Riemannian, the reference frame is (locally) inertial, and no any external field (geometric treatment);

c) spacetime is Euclidean in a global inertial frame, but the reference frame of the observer is accelerated and no external field (kinematic treatment).

After analyzing these situations in general relativity, the geometric theory of gravitation has been established. Analogously, we can conclude, that the quantum 
16 Zakir Z. (2009) Theoretical Physics, Astrophysics and Cosmology, 4, 10; TPAC: 3200-014 v2.

principle of equivalence allows us to justify the stochastic geometrical version of quantum mechanics.

Thus, quantum mechanics is nothing but as the stochastic geometry of spacetime and this fact has a very important consequence as an explanation of gravitation as inhomogeneous quantum diffusion [4].

\section{References}

1. Nelson E. (1966) Phys. Rev. 150, 1079; (1967) Dynamical Theories of Brownian Motion, Princeton U. Pr.

2. Blanchard Ph., Combe Ph., Zheng W. (1987) Mathematical and Physical Aspects of Stochastic Mechanics. Lect. Not. Phys., 281, 171 p.

3. Israilov Z.Zakir (1987) A generalized principle of relativity as the foundation of the theory of stochastic space and time. Prep. PTI-43-87-FVE, Tashkent.

4. Zakir Z. (2009) Theor. Phys., Astrophys. and Cosmol., 4, No 1, 1; arXiv:hep-th/9812254.

5. Feynman R.P., Hibbs A.R. (1965) Quantum Mechanics and Path Integrals. McGraw-Hill Book, N.Y.

6. Guerra F., Morato L. (1983) Phys.Rev. D 27, p.1774-1786.

7. Guerra F, Marra R. (1983) Phys.Rev. D 28, p.1916.

8. Smolin L. (1986) Phys.Lett., 113A, No 8, p. 408. 